\title{
A multilevel study of the environmental determinants of swine ascariasis in England
}

\author{
Ângelo Joel Mendes ${ }^{\mathrm{a}, \mathrm{b}, \mathrm{c}, *}$, Ana Isabel Ribeiro ${ }^{\mathrm{d}}$, Milton Severo ${ }^{\mathrm{d}}$, João Niza-Ribeiro ${ }^{\mathrm{a}, \mathrm{d}}$

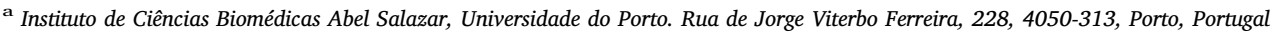 \\ b Faculdade de Medicina, Universidade do Porto, Alameda Prof. Hernâni Monteiro, 4200-319, Porto, Portugal \\ c Eville and Jones, Ltd., Century House, Thorpe Park, Leeds, LS15 8ZB, United Kingdom \\ d EPIUnit - Instituto de Saúde Pública, Universidade do Porto, Rua das Taipas, 135, 4050-600, Porto, Portugal
}

\section{A R T I C L E I N F O}

\section{Keywords:}

Ascariasis

Milk spots

Socioeconomic deprivation

Soil

Climate

Spatial analysis

\begin{abstract}
A B S T R A C T
Ascariasis is considered a common parasitosis of swine worldwide. The disease causes significant economic losses due to its effect on feed conversion ratio and liver condemnations at slaughter (liver milk spots).

This study aimed to characterise the between-farm and spatial variance in porcine ascariasis in England and to assess the association between the percentage of infected animals and potential environmental risk factors, including production system, socioeconomic deprivation, soil characteristics ( $\mathrm{pH}$, topsoil bulk density, topsoil organic matter, topsoil texture class, soil water regime, topsoil available water capacity, and elevation), and climatic conditions (relative humidity, air temperature, and rainfall) before slaughter.

Post-mortem inspection results were provided by the Food Standards Agency and comprised information about the number of rejected livers, the number of animals sent to slaughter and the production system. All farms were georeferenced based on the postcode, which allowed the assessment of the area index of socioeconomic deprivation and the extraction of soil and climatic characteristics available in different online databases. Under a multilevel framework with adjustment for spatial autocorrelation, a standard linear mixed model was fitted to estimate the association between these determinants and the percentage of infected animals.

From 2,513,973 English farmed pigs included in the study, 4.3\% had their livers rejected due to milk spots. The percentage of infected pigs per batch ranged from $0 \%$ to $100 \%$. The highest percentages were found in Surrey, East and West Sussex (8.9\%) and lowest in Leicestershire, Rutland and Northamptonshire (2.0\%). Significant associations were found at multivariable analysis between the proportion of infection and the number of animals sent to slaughter $(\beta=-0.005 ; 95 \% \mathrm{CI}=-0.005,-0.004)$, soil texture (peat compared to coarse textured soils; $\beta=-0.516 ; 95 \% \mathrm{CI}=-1.010,-0.063)$, relative humidity $(\beta=0.011 ; 95 \% \mathrm{CI}=0.006$, 0.015), mean temperature $(\beta=0.007 ; 95 \% \mathrm{CI}=0.003,0.012)$, and rainfall $(\beta=0.022 ; 95 \% \mathrm{CI}=0.004$, 0.037).

In conclusion, our findings suggest that ascariasis can be influenced by a complex network of environmental factors. Future research needs to acknowledge these intermingled relationships to guide the development and application of control measures by the industry.
\end{abstract}

\section{Introduction}

Ascariasis is the most common parasitosis of swine worldwide and is mainly caused by the helminth Ascaris suum (Stewart and Hoyt, 2013). The striking feature of this nematodiasis is the migration of the parasite through the liver, which affects animal welfare and productivity (Stewart and Hoyt, 2013). The pathological consequence is a chronic multifocal interstitial hepatitis that is usually asymptomatic. However, at post-mortem inspection, the liver will show multiple, spherical, and whitish foci, which are frequently named "milk spots," rendering it unfit for human consumption (Yoshihara et al., 1983). The detection of milk spots has been used as an indicator of Ascaris suum infection (Sanchez-Vazquez et al., 2012). According to Bernardo et al. (1990) the absence of milk spots is a reliable indicator of the absence of an established Ascaris infection. The authors reported the following characteristics for the use of milk spots as an indicator of the faecal evacuation of A. suum eggs: - sensitivity, 95.8\%; - specificity, $23.8 \%$; positive predictive value, $36.7 \%$; and, - negative predictive value, 92.5\%.

According to Roepstorff and Nansen (1994), a small percentage of

\footnotetext{
* Corresponding author at: Instituto de Ciências Biomédicas Abel Salazar. Universidade do Porto. Rua de Jorge Viterbo Ferreira, 228. 4050-313, Porto, Portugal.

E-mail address: ajmendes@icbas.up.pt (Â.J. Mendes).
} 
farms are free of the disease. Specific characteristics of the parasite may contribute to its ubiquity: (i) A. suum is a direct life cycle parasite, i.e., it does not involve an intermediate host; (ii) the adult female parasite is highly fecund (laying 1 million eggs, or more, daily); and, (iii) the eggs are especially resistant to environmental factors because of their complex and thick shell layers (Stewart and Hoyt, 2013).

A multi-national research study conducted in the Nordic countries found that $21.5 \%$ of the fatteners were infected (Roepstorff and Nansen, 1998). In Germany, ten out of 144 (7\%) swine farms randomly selected for a cross-sectional survey revealed ascariasis in sows (Gerwert et al., 2004). In England, a five-year monitoring programme found evidence of $A$. suum infection at slaughter (through the detection of milk spots) in $4.2 \%$ of 34,168 pigs inspected (Sanchez-Vazquez et al., 2012).

Production and management systems are known determinants of $A$. suum infection. The intensification of swine production systems has been linked to an overall increase in the prevalence of milk spots (Menzies et al., 1994). However, no differences in the prevalence of $A$. suum in pigs between intensive and extensive production systems have also been reported (Lai et al., 2011).

Since sanitation and improvement of hygiene practices represent the best methods to prevent and control the dissemination of infection in both humans and pigs, socioeconomic factors are also major determinants of the disease. The burden of human infection by A. lumbricoides is concentrated in low-income countries, where sanitation is poor, and the populations are more socioeconomically deprived (Brooker et al., 2006). For instance, (i) having less than four years of education, (ii) drinking untreated water, (iii) living in highly dense households, and (iv) having a salary below the minimum wage were associated with increased risk of human infection by A. lumbricoides in Brazil (Valencia et al., 2005). So, we must consider the possibility that these factors may play a role in the epidemiology of ascariasis in pigs. To the authors' knowledge, this has never been studied before.

In England and other countries, strong spatial inequalities in the distribution of swine ascariasis were observed, which suggests that the geographical location may act as a latent variable encapsulating several biogeophysical and socioeconomic factors (Roepstorff and Nansen, 1998; Sanchez-Vazquez et al., 2010). Accumulated evidence suggests that climatic and soil characteristics might also be possible determinants of the disease (Beaver, 1953; Arene, 1986; Kim et al., 2012; Schüle et al., 2014). For instance, it has been accepted for a long time that $A$. suum eggs do not develop under the temperature of $5{ }^{\circ} \mathrm{C}$ and the optimum temperature for cleavage seems to be between 25 and $30^{\circ} \mathrm{C}$ (Arene, 1986; Kim et al., 2012), which might explain the comparatively higher incidence of the disease in summer months (Sanchez-Vazquez et al., 2012; McCormick et al., 2013; Neumann et al., 2014). Water and oxygen availability also seem to influence the Ascaris life cycle and, consequently, the occurrence of the disease. A cross-sectional study that analysed the environmental determinants of $A$. lumbricoides infection in humans in Tanzania using remotely sensed data found a significant association between the level of rainfall and the prevalence of disease (Schüle et al., 2014). Previously, another study concluded that the "number of wet days" (rain days) could also be used as a predictor of Ascaris infection in humans (Gunawardena et al., 2004). In addition to the atmospheric conditions, several physicochemical characteristics of the soil, where part of the life cycle takes place, seem to play a role. Beaver (1953) showed that larvae in direct contact with sunlight and those unable to migrate vertically in compact soils, die quickly. A strong association between soil $\mathrm{pH}$ and the frequency of infective forms of intestinal parasites in topsoil samples collected from public squares has also been reported. Using Yule's Q coefficient, Sánchez Thevenet et al. (2004) found that the presence of intestinal parasites was relatively more common in soils with $\mathrm{pH}$ values between 7 and 9 .

The purpose of this study was then to characterise the between-farm and spatial variation in porcine ascariasis in England and to assess the association between the percentage of infection and previously mentioned potential environmental risk factors. These included (i) farming features (production system and size of the farm), (ii) the neighbourhood socioeconomic context (deprivation); and, (iii) the soil and climatic conditions (relative humidity, air temperature, rainfall, and soil characteristics - soil pH, topsoil bulk density, topsoil organic matter, topsoil texture class, soil water regime, topsoil available water capacity, and elevation).

\section{Material and methods}

\subsection{Post-mortem inspection data}

The number of infected animals was estimated using results of postmortem inspection. The post-mortem inspection data was provided by the Food Standards Agency (FSA), the non-ministerial body that enforces food safety regulations in food establishments across the United Kingdom. The data included post-mortem inspection results of $2014\left(1^{\text {st }}\right.$ January-31 ${ }^{\text {st }}$ December 2014) from 12 pig abattoirs located in different geographical regions across England. These abattoirs were chosen to provide a nationwide representation of farming settlements in England, as done by other authors (Sanchez-Vazquez et al., 2010; SanchezVazquez et al., 2011; Sanchez-Vazquez et al., 2012).

The dataset included the following variables: producer information (e.g. internal identification number, county parish holding $(\mathrm{CPH})$, and address), the slapmark/herdmark (i.e., a permanent ink mark with the herd number, applied to each shoulder of the pigs), the type of animals (fattening or cull pigs), the production system (born and reared under controlled housing conditions; born outdoors and reared under controlled housing conditions since weaning; and, born and reared outdoors), the date of slaughter, the number of pigs slaughtered per batch (throughput), and the number of livers rejected per batch due to milk spots. A total number of 21,895 registers, each corresponding to a batch of animals, was available.

A set of selection criteria was applied to meet the requirements of the study, clean the database from missing or misleading data, and improve the representativeness of the sample. The data entries were excluded if (1) the postcode was absent or poor in quality ( $n=1895$ batches), (2) the batches were from Irish, Scottish or Welsh farms $(\mathrm{n}=652)$, (3) less than five pigs per batch were sent to slaughter ( $n=276)$, (4) the batch was mixed (from markets and different farm origins) ( $n=712)$, (5) the batch was composed of cull pigs $(n=472)$, and, (6) the producer $\mathrm{CPH}$ was absent or incorrect $(\mathrm{n}=738)$. This process resulted in a database comprising 1463 farms, 17,150 batches, and 2,513,973 pigs. Throughout the selection process, no significant spatial differences were observed; except when excluding farms that were located in Northern Ireland, Scotland, and Wales.

Farms were georeferenced based on the postcode of the $\mathrm{CPH}$ using ArcGIS (version 10.4.1 - ESRI, Redlands, CA), which then allowed us to characterise geographic patterns and attribute a set of contextual variables (described ahead) to each farm.

\subsection{Covariates}

We included covariates from three key groups of determinants farm production and management practices, soil characteristics, and climatic conditions before slaughter.

\subsubsection{Production and management conditions of the farm and socioeconomic context}

Although a variable about the production system of each farm was available (born and reared under controlled housing conditions; born outdoors and reared under controlled housing conditions since weaning; and, born and reared outdoors), it was incomplete in $70.9 \%$ of the registers. Thus, in the absence of detailed and complete information about production and management conditions, the throughput (number of animals sent to slaughter) was used as a representation of farm size.

The characterisation of the socioeconomic context of the farm's 
geographical area was based on the 2015 English Index of Multiple Deprivation (EIMD15). The EIMD15 is the official measure of relative deprivation in England and ranks every small area from 1 (most deprived area) to 32,844 (least deprived area). It was produced considering seven different domains with respective weights (income $-22.5 \%$; employment $-22.5 \%$; education, skills and training $-13.5 \%$; health and disability $-13.5 \%$; crime $-9.3 \%$; barriers to housing and services $-9.3 \%$; and, living environment $-9.3 \%$ ). The methodology used to construct the EIMD15 is fully described in its technical report (Department for Communities and Local Government, 2015). The ranked areas were those defined by the 2011 Lower layer Super Output Area (LSOA) boundaries, which delimitate small homogenous areas of relatively equal size containing approximately 1500 people (Office for National Statistics, 2011). A categorisation of the deciles was made for further statistical analysis as follows: - deciles 1-4 (most deprived); deciles 5 and 6 (medium deprived); and, - deciles 7-10 (least deprived).

\subsubsection{Soil characteristics}

The soil characteristics (soil $\mathrm{pH}$, topsoil bulk density, topsoil organic matter, topsoil texture class, soil water regime, topsoil available water capacity, and elevation above sea level) were retrieved from several open data services. The metadata for each biogeophysical variable is provided in Table 1.

The soil $\mathrm{pH}$ measures the acidity or alkalinity level of the soil. It reflects several physical and chemical conditions of the environment and corresponds to the negative decimal logarithm of the hydrogen ion $(\mathrm{H}+)$ concentration in soil solution (Chesworth, 2007).

The topsoil bulk density, a measure of the compaction of the first $30 \mathrm{~cm}$ of soil depth, is the quotient of the soil mass and its total volume (Shukla, 2013). The more porous the soil, the lower the value of bulk density.

The topsoil organic matter is a measure of the amount of organic matter in the soil, which is mostly produced by vegetation death and decomposition by soil microorganisms (Lal, 2006).

Texture conveys an idea of the physical properties of the soil according to the proportion of three fractions (sand, silt and clay). Soil texture is always expressed into classes that reflect the relative amount of each fraction. Specifically, the topsoil texture class is the result of the proportion of sand $(2-0.02 \mathrm{~mm})$, silt $(0.02-0.002 \mathrm{~mm})$ and clay
( $<0.002 \mathrm{~mm})$ on the soil, as follows: - coarse $(18 \%<$ clay and $>65 \%$ sand); - medium $(18 \%<$ clay $<35 \%$ and $\geq 15 \%$ sand, or $18 \%<$ clay and $15 \%<$ sand $<65 \%$ ); - medium fine $(<35 \%$ clay and $<15 \%$ sand); - fine $(35 \%<$ clay $<60 \%)$; - very fine (clay $>60 \%)$; and, - no mineral texture (peat soils).

The soil water regime indicates the amount of the water kept in the soil. It is an annual estimation of the moisture conditions prevailing in the soil profile (Lal, 2006), as follows: - not wet within $80 \mathrm{~cm}$ for over 3 months, nor wet within $40 \mathrm{~cm}$ for over 1 month ("dry"); - wet within $80 \mathrm{~cm}$ for 3-6 months, but not wet within $40 \mathrm{~cm}$ for over 1 month ("least wet"); - wet within $80 \mathrm{~cm}$ for over 6 months, but not wet within $40 \mathrm{~cm}$ for over 11 months ("moderately wet"); and, - wet within $40 \mathrm{~cm}$ depth for over 11 months ("most wet"). The topsoil available water capacity measures the amount of water (in millimetres of water per metre of soil depth) retained by the soil profile and has been classified as follows: - low ( $<100 \mathrm{~mm} / \mathrm{m})$; - medium $(100-140 \mathrm{~mm} / \mathrm{m})$; - high $(140-190 \mathrm{~mm} / \mathrm{m})$; and, - very high ( $>190 \mathrm{~mm} / \mathrm{m})$. Finally, the elevation is a measure of the height above sea level, in metres.

\subsubsection{Climate conditions before slaughter}

The climatic data for 2014 (monthly relative humidity; daily air temperatures - minimum and maximum; and, daily rainfall totals) was acquired from the Met Office, the United Kingdom's national weather service. This database comprised $5 \mathrm{~km}$ grid squares masked to the UK, and further clipped to England. The values were cell-centred estimations and were imported by the authors as ASCII files into ArcGIS. Then, these variables were averaged for the 44 days before slaughter at each farm location because this is the period with the potential influence on the post-mortem inspection findings (Stewart and Hoyt, 2013). The life cycle of A. suum includes (i) a period of approximately 30 days when embryonated eggs are excreted into the environment, develop into L2 (second stage larvae) and L3 (infective and third stage larvae), and, (ii) a period of nearly seven days when L3 are ingested, hatch in the stomach, reach the intestine, penetrate the intestinal mucosa, and migrate to the liver where milk spots develop (Stewart and Hoyt, 2013). Another seven days were added to reflect as much exposure of the animals to potential infective larvae as possible. The number of days with (i) minimum air temperature below $5{ }^{\circ} \mathrm{C}$ (Stewart and Hoyt, 2013) and (ii) recorded rainfall ("wet days") were also computed to assess the influence of potential thresholds (Gunawardena et al., 2004).

Table 1

Metadata for biogeophysical variables.

\begin{tabular}{|c|c|c|c|c|c|c|c|}
\hline Product name & Variable & Unit & Format & Version/ release date & Resolution & Projection & Original geographical coverage \\
\hline Soil pH in Europe ${ }^{a}$ & Soil pH & $\mathrm{N} / \mathrm{A}$ & Raster (.tif) & 2010 & $1 \mathrm{~km}$ & ETRS89 & $\begin{array}{l}\text { EU-25 + Norway, Switzerland, Croatia, } \\
\text { Albania }\end{array}$ \\
\hline $\begin{array}{l}\text { EFSA Data and PERSAM } \\
\text { software tool }\end{array}$ & $\begin{array}{l}\text { Topsoil bulk density } \\
\text { Topsoil organic matter } \\
\text { Topsoil texture class }\end{array}$ & $\begin{array}{l}\mathrm{kg} / \mathrm{m}^{3} \\
\% \\
\mathrm{~N} / \mathrm{A}\end{array}$ & $\begin{array}{l}\text { Raster } \\
\text { (ASCII) }\end{array}$ & Nov 2012 & $1 \mathrm{~km}$ & ETRS89 & $\begin{array}{l}\text { EU-27 + Balkan countries, Switzerland and } \\
\text { Norway }\end{array}$ \\
\hline European Soil Database $^{c}$ & $\begin{array}{l}\text { Soil water regime } \\
\text { Topsoil available water } \\
\text { capacity }\end{array}$ & N/A & Raster (.tif) & $\begin{array}{l}\text { V2.0, } 2006 \\
25 \text { Sep } 2001\end{array}$ & $1 \mathrm{~km}$ & ETRS89 & $\begin{array}{l}\text { EU-27 + Balkan countries, Switzerland, } \\
\text { Norway, Ukraine and Russia }\end{array}$ \\
\hline OS Terrain $50^{\mathrm{d}}$ & Elevation & $\mathrm{m}$ & $\begin{array}{l}\text { Raster } \\
\text { (ASCII) }\end{array}$ & Jul 2015 & $50 \mathrm{~m}$ & BNG & Great Britain \\
\hline $\mathrm{UKCPO9}^{\mathrm{e}}$ & $\begin{array}{l}\text { Air temperatures } \\
\text { Rainfall totals } \\
\text { Relative humidity }\end{array}$ & $\begin{array}{l}{ }^{\circ} \mathrm{C} \\
\mathrm{mm} \\
\%\end{array}$ & $\begin{array}{l}\text { Raster } \\
\text { (ASCII) }\end{array}$ & $\begin{array}{l}\text { Data for } 2014 \text { released } \\
\text { on } 18 \text { Dec } 2015\end{array}$ & $\begin{array}{l}5 \mathrm{~km} \text {, daily } \\
5 \mathrm{~km} \text {, daily } \\
5 \mathrm{~km} \text {, monthly }\end{array}$ & BNG & United Kingdom \\
\hline
\end{tabular}

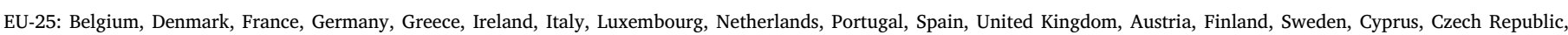

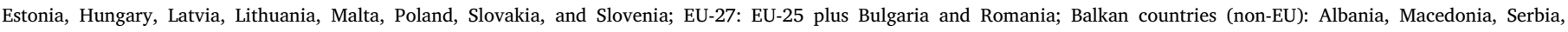

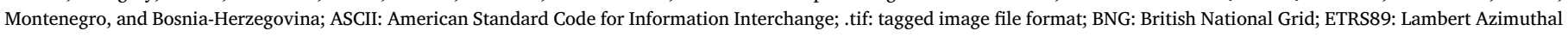
Equal Area; N/A: non-applicable.

${ }^{a}$ European Soil Data Centre (ESDAC) (2010). Soil pH in Europe. Retrieved from http://esdac.jrc.ec.europa.eu/content/soil-ph-europe on 25 th February 2016.

b European Soil Data Centre (ESDAC) (2012). European Food Safety Authority (EFSA) Data and PERSAM software tool. Retrieved from http://esdac.jrc.ec.europa.eu/content/ european-food-safety-authority-efsa-data-persam-software-tool on 25th February 2016.

${ }^{c}$ European Soil Data Centre (ESDAC) (2001). European Soil Database v2.0 (vector and attribute data). Retrieved from http://esdac.jrc.ec.europa.eu/content/european-soil-databasev20-vector-and-attribute-data on 25th February 2016.

d Ordnance Survey (2015). OS Terrain 50. Retrieved from https://www.ordnancesurvey.co.uk/business-and-government/products/terrain-50.html on 12 th November 2015.

${ }^{\mathrm{e}}$ Met Office (2015). UKCP09: Daily data sets. Obtained under service request on 5th December 2015. 


\subsection{Statistical analysis}

The proportion of infected animals per batch was calculated dividing the number of rejected livers (due to the presence of milk spots) by the number of livers inspected per batch. The proportion of infected batches (number of batches with at least one liver rejected due to milk spots divided by the total number of batches inspected) and farms (number of farms with at least one batch infected divided by the total number of farms) were also calculated. Descriptive statistics were calculated for categorical (counts and proportions) and numerical variables (median and interquartile range, IQR). The assessment of normality, homoscedasticity, collinearity and spatial autocorrelation was performed as part of the modelling assumptions and baseline building tests.

Choropleth maps were built for spatial visualisation of the proportion of infected animals per farm. This procedure only included areas of NUTSII (level two of the Nomenclature of Territorial Units for Statistics) with at least five farms to minimise the random fluctuations associated with dealing with small numbers.

The assessment of associations between the suggested risk factors and the percentage of infected animals was performed using a standard Gaussian multilevel linear mixed model (LMM) with non-parametric spatial terms (smoothing splines for the longitude and latitude of the farm). Since the distribution of the percentage of infected pigs per batch was right-skewed, a log-transformation was applied. A three-level structure of data was also considered, i.e. the inspected batches (first level) were nested within the farm of provenance (second level) and further nested within each abattoir (third level), where specific official protocols might have had some influence on inspection results. A stepwise procedure was conducted as follows: i) firstly, an univariable multilevel LMM analysis, with spatial non-parametric terms and random effects, was performed to evaluate the association between each risk determinant and the percentage of porcine ascariasis per batch; ii) secondly, three successive models were built from a random effects and spatial effects model (model 0), adding production and management practices (block 1), soil characteristics (block 2), and climatic conditions before slaughter (block 3). So, the null model (model 0) was the starting point and included only spatial non-parametric terms and random effects, the model 1 consisted of the null model plus production and management practices, the model 2 consisted of the model 1 plus soil characteristics, and the model 3 consisted of the model 2 plus climatic conditions before slaughter. The workflow of data analysis is shown in Fig. 1. The variables that showed strong and significant autocorrelation (Spearman's rho larger than 0.8) were excluded from the stepwise model building procedure. We kept those with smaller $p$-value and that yield better model fitting. Thus, the organic matter content of the soil, the water capacity, and the climatic variables other than mean temperature, relative humidity and rainfall were omitted from the multivariable models. Complementary, we computed the intraclass correlation coefficient (ICC), a measure of clustering correlation that expresses the proportion of the total variance occurring at each level (Merlo et al., 2005). Furthermore, this modelling sequence allowed the assessment of the proportional change in variance caused by the inclusion of each batch of covariates using the previous model variance as a reference.

The multi-level analysis was conducted in R (package lme4; Bates et al., 2015) and an ordinary nonparametric bootstrap with 500 bootstrap replicates was used to compute confidence intervals of LMM estimates, including variance components (package boot; Canty and Ripley, 2016). The significance level was set at 0.05 .

\subsection{Ethics statement}

The present study used official information in accordance with FSA's Privacy Statement and the exemption in section 31 of the Data Protection Act (1998). The data supplied by FSA operations group was extracted exclusively from the INNOVA system. The authors were aware of and have undertaken to comply with the requirements of the Data Protection Act (1998), Freedom of Information Act (2000), and Environmental Information Regulations (2004). The results of this research study were to be published in such a way that information relating to any particular land, business or person could not be inferred from it.

\section{Results}

\subsection{Descriptive statistics: spatial distribution and differences between farms and abattoirs}

From the 2,513,973 pigs slaughtered, 108,667 (4.3\%) had their livers rejected due to milk spots. The median percentage of infected pigs per batch was $1.3 \%$ (IQR $=0.5-4.0)$, ranging from zero $(n=2448)$ to $100 \%(\mathrm{n}=56)$. The median percentage of infected pigs per farm was $1.6 \%(\mathrm{IQR}=0.9-3.7)$, ranging from zero $(\mathrm{n}=161)$ to $100 \%(\mathrm{n}=7)$.

The distribution of the percentage of infected animals per NUTSII is shown in Fig. 2. Among the areas with, at least, five farms assessed $(n=23)$, the proportion of rejected livers ranged from $2.0 \%$ in Leicestershire, Rutland and Northamptonshire, where 1085 livers were rejected from 54,736 pigs (30 farms) to $8.9 \%$ in Surrey and Sussex (East and West), where 233 livers were rejected from 2609 pigs (7 farms).

The proportion of rejected livers per batch was not independent of the abattoir. Table 2 shows the proportion of infected pigs per abattoir. In 11 out of 12 abattoirs, the percentage of swine ascariasis ranged from $2.2 \%$ (abattoir $\mathrm{J}$ and $\mathrm{K}$ ) to $8.5 \%$ (abattoir $\mathrm{G}$ ). In abattoir I, this percentage was significantly higher $(21.1 \%)$. This abattoir was also the smallest one, with fewer pigs slaughtered $(11,490)$, while the abattoir B had the highest throughput $(814,313)$.

The data selection procedure did not affect these results greatly as only $17.36 \%$ of the animals included in the initial database were excluded. Through this process, the percentage of livers with milk spots decreased from 4.56 to 4.32 . The abattoirs where a higher proportion of animals were excluded were the $\mathrm{G}(77.86 \%)$ and I $(50.84 \%)$. The abattoir $\mathrm{G}$ slaughters a great proportion of cull pigs. At the abattoir I, $20 \%$ and $19 \%$ of the slaughtered batches had a missing postcode and comprised less than five pigs, respectively.

\subsection{Descriptive statistics: frequency of infection according to covariates}

The distribution of the inspected animals and infected animals according to the variables related to production and management practices, soil characteristics and climatic conditions before slaughter is shown in Table 3. We observed that the proportion of infected animals varied significantly according to the levels (tertiles) of the covariates considered. For instance, the proportion of infected animals was higher in smaller batches, produced indoors, from farms located in more deprived areas, more elevated areas, in areas of higher relative humidity, and rainfall, of lower soil $\mathrm{pH}$, of lower percentage of soil organic matter, and of higher soil bulk density.

The spatial distribution of the most important covariates is shown in Figs. S1-S10 (Supplementary material).

\subsection{Statistical modelling: associations between the percentage of infected animals and the covariates}

\subsubsection{Univariable analysis}

At univariable analysis, the proportion of porcine ascariasis per batch was significantly associated with the throughput, area socioeconomic deprivation, soil texture, water capacity, relative humidity, and rainfall. No association was observed with the water regime, $\mathrm{pH}$, bulk density, elevation, organic matter content, mean temperature, minimum temperature, maximum temperature, number of days with minimum temperature below $5{ }^{\circ} \mathrm{C}$, and "wet days". With an increase in the number of animals sent to slaughter, the proportion of porcine ascariasis decreased significantly $(\beta=-0.005$; 

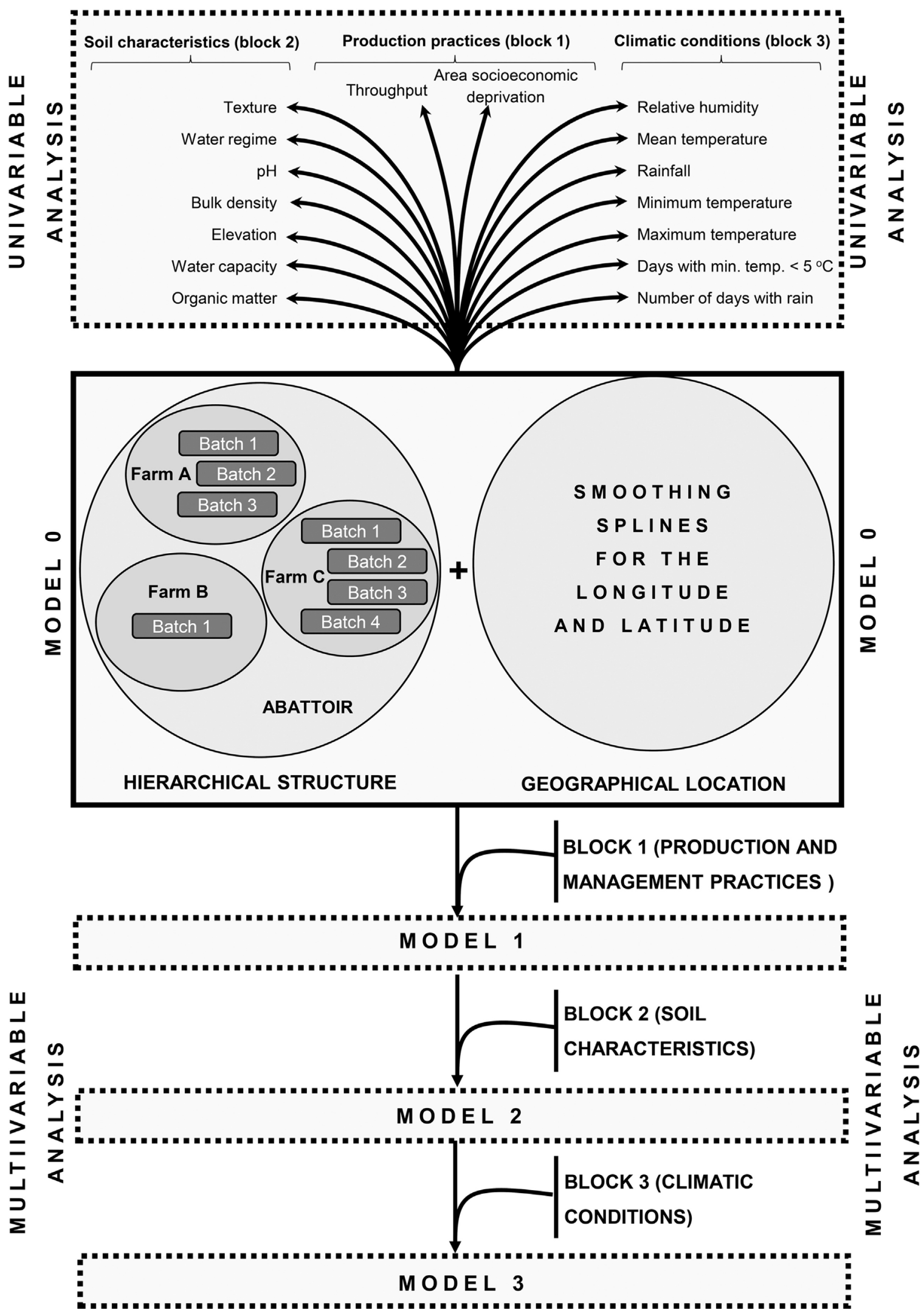

Fig. 1. Workflow of data analysis.

$95 \% \mathrm{CI}=-0.005,-0.004)$. The farms located in areas with the best ranks of socioeconomic deprivation had a significantly lower proportion of porcine ascariasis (least deprived compared to most deprived areas; $\beta=-0.210$; $95 \% \mathrm{CI}=-0.417,-0.030$ ). The farms located in coarse soils appear to have a significantly higher percentage of infected animals compared to those located in medium texture class $(\beta=-0.187 ; 95 \% \mathrm{CI}=-0.359,-0.018)$ and peat soils $(\beta=-0.451 ; 95 \% \mathrm{CI}=-0.897,-0.030)$. Finally, we observed a positive association between the percentage of infected animals per 


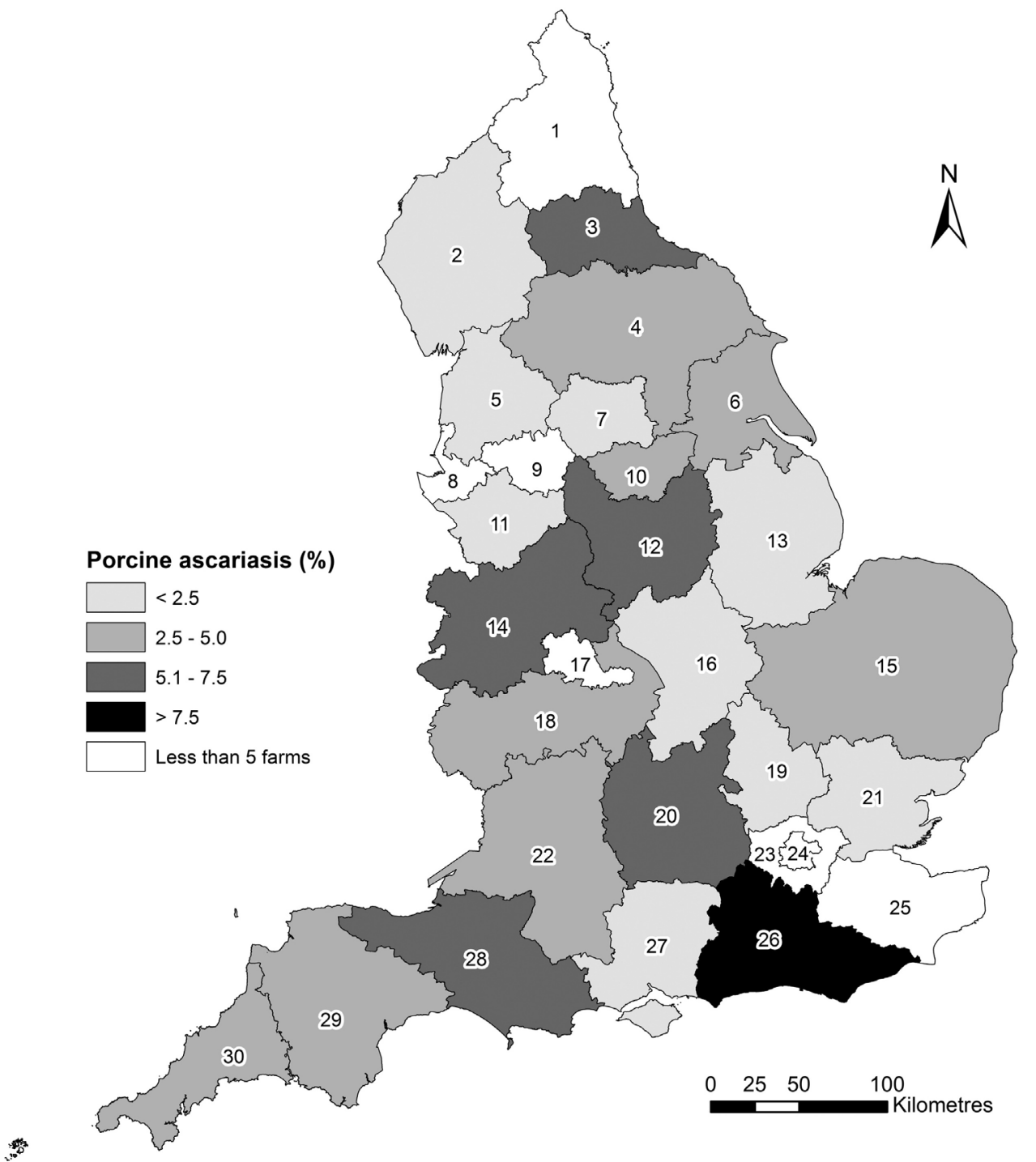

Fig. 2. Choropleth map showing the spatial distribution of porcine ascariasis according to the percentage of rejected livers due to milk spots per geographic area. (C) EuroGeographics for the administrative boundaries, Eurostat. Legend: 1. Northumberland and Tyne and Wear; 2. Cumbria; 3. Tees Valley and Durham; 4. North Yorkshire; 5. Lancashire; 6. East Riding and North Lincolnshire; 7. West Yorkshire; 8. Merseyside; 9. Greater Manchester; 10. South Yorkshire; 11. Cheshire; 12. Derbyshire and Nottinghamshire; 13. Lincolnshire; 14. Shropshire and Staffordshire; 15. East Anglia; 16. Leicestershire, Rutland and Northamptonshire; 17. West Midlands; 18. Herefordshire, Worcestershire and Warwickshire; 19. Bedfordshire and Hertfordshire; 20. Berkshire, Buckinghamshire and Oxfordshire; 21. Essex; 22. Gloucestershire, Wiltshire and North Somerset; 23. Outer London; 24. Inner London; 25. Kent; 26. Surrey, East and West Sussex; 27. Hampshire and Isle of Wight; 28. Dorset and Somerset; 29. Devon; and, 30. Cornwall and Isles of Scilly.
Table 2

Descriptive statistics of the outcome variable.

\begin{tabular}{lll}
\hline Variable & & Count (\%) \\
\hline Infected pigs & & $108,667(4.3)$ \\
Infected batches & & $14,724(85.9)$ \\
Infected farms & & $1302(89.0)$ \\
Infected pigs by abattoir & A & $9118(5.9)$ \\
& B & $31,554(3.9)$ \\
& C & $6740(2.5)$ \\
D & $4694(6.5)$ \\
& E & $1488(4.4)$ \\
& F & $12,983(4.9)$ \\
& G & $3017(8.5)$ \\
& H & $21,188(7.2)$ \\
& I & $2426(21.1)$ \\
J & $4811(2.2)$ \\
& K & $2926(2.3)$ \\
& L & $7722(3.5)$ \\
\hline
\end{tabular}

batch and both the relative humidity $(\beta=0.007 ; 95 \% \mathrm{CI}=0.003,0.010)$ and rainfall $(\beta=0.024 ; 95 \% \mathrm{CI}=0.008,0.041)$ (Table 4$)$.

\subsubsection{Multivariable analysis}

The stepwise inclusion of production and management practices (model 1), soil characteristics (model 2), and climatic conditions (model 3) to the null model kept most of the associations observed at univariable analysis. However, the significant relationship observed between the area socioeconomic deprivation and the percentage of infected animals per batch was lost when the variable throughput was added. This association would be kept significant in the final model if the spatial structure of the data had not been considered (model A; $\beta=-0.220 ; 95 \% \mathrm{CI}=-0.425,-0.020$ ). The impact of soil texture was also attenuated when the model was adjusted for the remaining variables of block 1 and 2. Though, peat soils seemed to be associated with a lower percentage of disease when compared with coarse soils ( $\beta=-0.525 ; 95 \% \mathrm{CI}=-1.022,-0.056)$. Finally, the third model, with the three building blocks, revealed a significant association with the mean air temperature. Warmer periods pre-slaughter were associated with higher percentage of infected animals per batch $(\beta=0.007$; $95 \% \mathrm{CI}=0.003,0.012$ ) (Table 4). A choropleth map showing the predicted spatial distribution of porcine ascariasis according to the model 3 is available in Fig. S11 (Supplementary material).

The variance components of the models are shown in Table 5. The between-farm and between-abattoir variances observed were 1.420 $(95 \% \mathrm{CI}=1.297,1.525)$ and $0.394(95 \% \mathrm{CI}=0.127,0.779)$, respectively. The ICC revealed that $55.7 \%(95 \% \mathrm{CI}=49.9,60.5)$ and $15.4 \%$ $(95 \% \mathrm{CI}=5.9,25.5)$ of the variance was at farm and abattoir level, respectively. We observed that $6.0 \%(95 \% \mathrm{CI}=5.3,7.3)$ of the total between-farm variance and $35.3 \%(95 \% \mathrm{CI}=27.3,41.5)$ of the total 
Table 3

Number and percentage of animals inspected and infected according to the variables related to production and management practices, soil characteristics and climatic conditions before slaughter.

\begin{tabular}{|c|c|c|}
\hline Variable & No. animals (\%) & No. infected animals (\%) \\
\hline \multicolumn{3}{|c|}{ Production and management practices } \\
\hline \multicolumn{3}{|l|}{ Production system } \\
\hline Outdoors & $782(0.0)$ & $16(2.0)$ \\
\hline Mixed & $18,775(0.7)$ & $778(4.1)$ \\
\hline Indoors & $658,165(26.2)$ & $34,784(5.3)$ \\
\hline Missing & $1,836,251(73.0)$ & $73,089(4.0)$ \\
\hline \multicolumn{3}{|l|}{ Throughput } \\
\hline $5-118$ & $449,183(17.9)$ & $34,513(7.7)$ \\
\hline $119-174$ & $510,496(20.3)$ & $23,528(4.6)$ \\
\hline $175-661$ & $1,554,294(61.8)$ & $50,626(3.3)$ \\
\hline \multicolumn{3}{|c|}{ Area socioeconomic deprivation } \\
\hline Least deprived & $901,033(35.8)$ & $26,304(2.9)$ \\
\hline Medium deprived & $1,129,541(44.9)$ & $56,504(5.0)$ \\
\hline Most deprived & $483,399(19.2)$ & $25,859(5.3)$ \\
\hline \multicolumn{3}{|l|}{ Soil characteristics } \\
\hline \multicolumn{3}{|l|}{ Texture } \\
\hline Coarse & $648,898(25.8)$ & $32,992(5.1)$ \\
\hline Medium & $1,320,653(52.5)$ & $51,495(3.9)$ \\
\hline Medium fine & $256,036(10.2)$ & $13,424(5.2)$ \\
\hline Fine & $228,929(9.1)$ & $9700(4.2)$ \\
\hline Peat soils & $59,457(2.4)$ & $1056(1.8)$ \\
\hline \multicolumn{3}{|l|}{ Water regime } \\
\hline Dry & $1,121,439(44.6)$ & $51,957(4.6)$ \\
\hline Least wet & $2881(0.1)$ & $86(3.0)$ \\
\hline Moderately wet & $696,567(27.7)$ & $34,628(5.0)$ \\
\hline Most wet & $663,255(26.4)$ & $21,383(3.2)$ \\
\hline Missing & $29,831(1.2)$ & $613(2.1)$ \\
\hline \multicolumn{3}{|l|}{ Water capacity } \\
\hline Medium & $587,128(23.4)$ & $29,433(5.0)$ \\
\hline High & $1,581,521(62.9)$ & $64,141(4.1)$ \\
\hline Very high & $256,036(10.2)$ & $13,424(5.2)$ \\
\hline Missing & $89,288(3.6)$ & $1669(1.9)$ \\
\hline \multicolumn{3}{|l|}{ Soil pH } \\
\hline $3.56-4.69$ & $943,984(37.5)$ & $39,274(4.2)$ \\
\hline $4.70-5.20$ & $678,270(27.0)$ & $38,241(5.6)$ \\
\hline $5.21-6.25$ & $835,232(33.2)$ & $30,209(3.6)$ \\
\hline Missing & $56,487(2.2)$ & $943(1.7)$ \\
\hline \multicolumn{3}{|l|}{ Bulk density $\left(\mathrm{kg} / \mathrm{m}^{3}\right)$} \\
\hline $217-1165$ & $916,545(36.5)$ & $38,514(4.2)$ \\
\hline 1169-1299 & $584,453(23.2)$ & $23,863(4.1)$ \\
\hline $1300-1503$ & $1,012,975(40.3)$ & $46,290(4.6)$ \\
\hline \multicolumn{3}{|l|}{ Organic matter (\%) } \\
\hline $1-2$ & $727,318(28.9)$ & 35,589 (4.9) \\
\hline $3-6$ & $1,279,847(50.9)$ & $53,152(4.2)$ \\
\hline $7-72$ & $506,808(20.2)$ & $19,926(3.9)$ \\
\hline \multicolumn{3}{|l|}{ Elevation (m) } \\
\hline$-8-43$ & $1,103,908(43.9)$ & $46,314(4.2)$ \\
\hline $44-97$ & $733,409(29.2)$ & $25,922(3.5)$ \\
\hline $99-415$ & $633,211(25.2)$ & $35,493(5.6)$ \\
\hline Missing & $43,445(1.7)$ & $938(2.2)$ \\
\hline \multicolumn{3}{|c|}{ Climatic conditions before slaughter } \\
\hline \multicolumn{3}{|c|}{ Relative humidity (\%) } \\
\hline $70.8-80.7$ & $961,937(38.3)$ & $38,503(4.0)$ \\
\hline $80.8-84.4$ & $616,251(24.5)$ & $26,180(4,2)$ \\
\hline $84.5-95.6$ & $935,580(37.2)$ & $43,984(4.7)$ \\
\hline Missing & $205(0.0)$ & $0(0.0)$ \\
\hline Minimum temperatu & & \\
\hline $0.45-5.23$ & $956,876(38.1)$ & $41,775(4.4)$ \\
\hline $5.25-8.14$ & $485,629(19.3)$ & $20,948(4.3)$ \\
\hline $8.16-14.43$ & $1,070,166(42.6)$ & $45,942(4.3)$ \\
\hline Missing & $1302(0.1)$ & $2(0.2)$ \\
\hline Maximum temperatu & & \\
\hline $4.36-12.45$ & $930,024(37.0)$ & $42,218(4.5)$ \\
\hline $12.48-16.50$ & $591,738(23.5)$ & $23,956(4.0)$ \\
\hline $16.52-23.77$ & $990,909(39.4)$ & $42,491(4.3)$ \\
\hline Missing & $1302(0.1)$ & $2(0.2)$ \\
\hline
\end{tabular}

Table 3 (continued)

\begin{tabular}{lll}
\hline Variable & No. animals $(\%)$ & No. infected animals (\%) \\
\hline & & \\
Mean temperature $\left({ }^{\circ} \mathrm{C}\right)$ & & \\
$2.48-8.85$ & $942,856(37.5)$ & $41,997(4.5)$ \\
$8.86-12.31$ & $541,080(21.5)$ & $22,458(4.2)$ \\
$12.32-18.74$ & $1,028,735(40.9)$ & $44,210(4.3)$ \\
Missing & $1302(0.1)$ & $2(0.2)$ \\
Number of days with minimum temperature $\left({ }^{\circ} \mathrm{C}\right)$ & $<5$ \\
$0-8$ & $1,240,647(49.4)$ & $\mathbf{5 3 , 6 5 4}(4.3)$ \\
$9-20$ & $405,486(16.1)$ & $16,787(4.1)$ \\
$21-44$ & $\mathbf{8 6 6 , 5 3 8}(34.5)$ & $38,224(4.4)$ \\
Missing & $1302(0.1)$ & $2(0.2)$ \\
Rainfall (mm) & & \\
$0.2-1.5$ & $\mathbf{8 4 9 , 3 6 1 ( 3 3 . 8 )}$ & $33,959(4.0)$ \\
$1.6-2.3$ & $1,009,652(40.2)$ & $46,283(4.6)$ \\
$2.4-10.4$ & $653,658(26.0)$ & $28,423(4.3)$ \\
Missing & $1302(0.1)$ & $2(0.2)$ \\
Days with rain (“wet days") & & \\
$3-14$ & $906,035(36.0)$ & $38,012(4.2)$ \\
$15-19$ & $907,882(36.1)$ & $39,820(4.4)$ \\
$20-43$ & $698,754(27.8)$ & $30,833(4.4)$ \\
Missing & $1302(0.1)$ & $2(0.2)$ \\
\hline
\end{tabular}

Note: continuous variables are categorised into tertiles. The $p$-value of the chi-square test is lower than 0.05 for all the covariates considered. The percentage on the column "No. animals (\%)" has the total number of animals inspected as denominator. The percentage on the column "No. infected animals (\%)" has the number of animals inspected on each category as denominator.

between-abattoir variance was explained by the block 1 (production and management practices - throughput and area socioeconomic deprivation). Adding the block 2 (soils characteristics; model 2) and 3 (climatic conditions; model 3) did not affect the variance components significantly.

\section{Discussion}

\subsection{Study findings}

This study was carried out to characterise the between-farm and spatial variation in porcine ascariasis in England and to assess the association between the percentage of infection and potential environmental risk factors, including biogeophysical (relative humidity, air temperature, rainfall, and soil characteristics - $\mathrm{pH}$, topsoil bulk density, topsoil organic matter, elevation, topsoil texture class, soil water regime, and topsoil available water capacity) and farming conditions (production system, size of the farm, and area socioeconomic context).

The overall percentage of liver milk spots in 2,513,973 pigs slaughtered was $4.3 \%$. This result is similar to the $4.2 \%$ observed by Sanchez-Vazquez et al. (2012), who conducted a five-year monitoring programme involving 34,168 pigs in England. Previously, SanchezVazquez et al. (2010) had reported a prevalence of $4.4 \%$ of porcine milk spots while investigating the effects of husbandry practices, geographical locations and seasonal patterns in Great Britain. According to these authors, the risk of milk spots was significantly higher in pigs farmed in the north (northeast and northwest England, York and the Humber) and southwest (west Midlands, Wales and southwest England) of Great Britain, compared to those farmed in the southeast (east Midlands, east and southeast England). In our study, using this regional classification, we also observed a higher percentage of milk spot livers in pigs farmed in the southwest $(4.8 \%$; $n=305,742$; Wales not included) compared to the southeast $(4.4 \%$; $n=867,017)$, but a lower percentage in the north $(4.2 \% ; n=1,341,214)$.

From the 1463 farms enrolled in this study, 1302 (89.0\%) had at least one liver rejected due to milk spots. This result seems to corroborate what had been acknowledged by Roepstorff and Nansen (1994): a small percentage of farms are free of the disease. However, our 


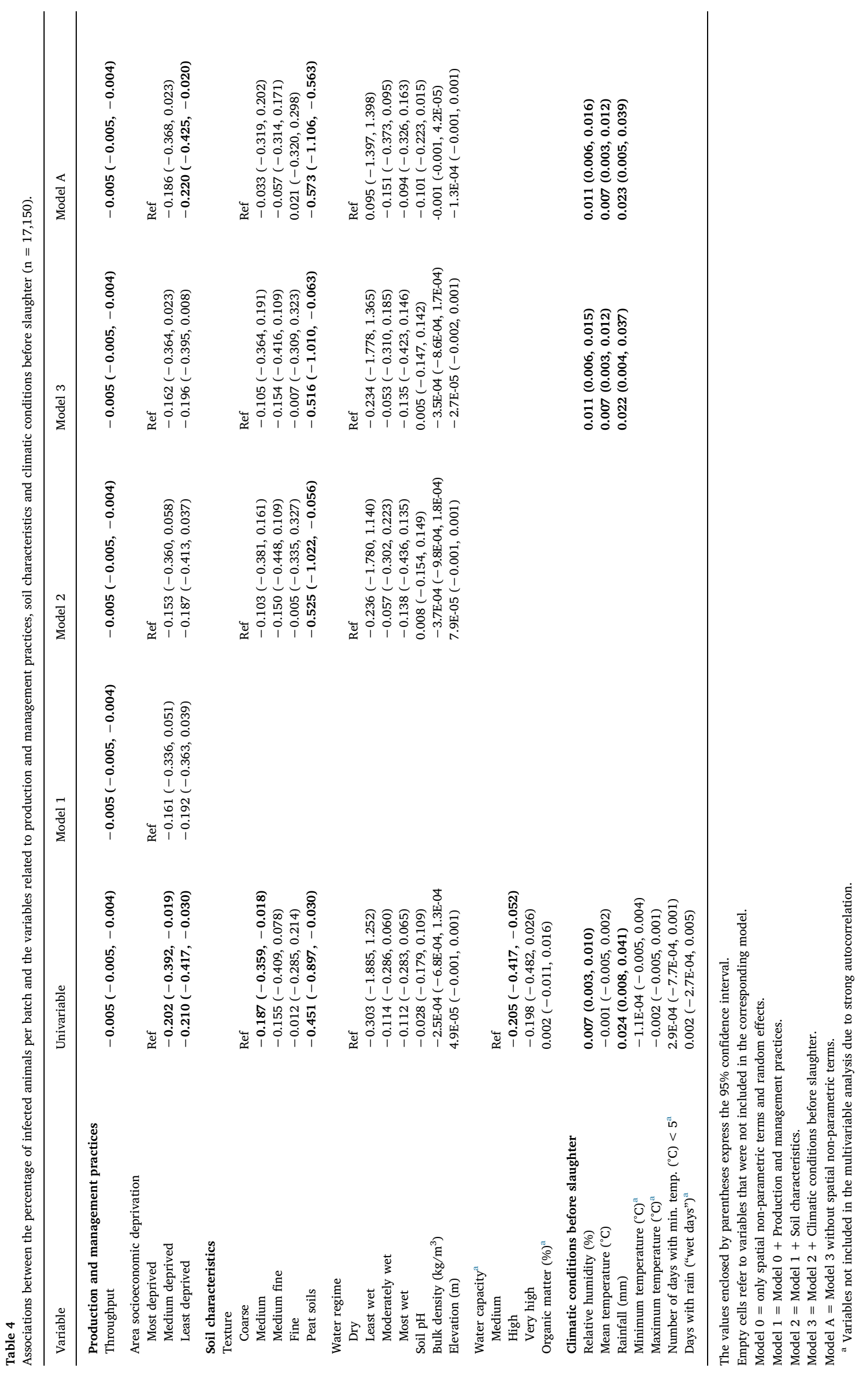


Table 5

Variance components at the farm and abattoir level of the four fitted models $(n=17,150)$.

\begin{tabular}{|c|c|c|c|c|}
\hline & Model 0 & Model 1 & Model 2 & Model 3 \\
\hline \multicolumn{5}{|l|}{ Variance } \\
\hline Farm level & $1.420(1.297,1.525)$ & $1.334(1.243,1.458)$ & $1.335(1.226,1.448)$ & $1.329(1.220,1.454)$ \\
\hline Abattoir level & $0.394(0.127,0.779)$ & $0.255(0.086,0.564)$ & $0.244(0.081,0.491)$ & $0.247(0.080,0.524)$ \\
\hline Residual & $0.737(0.720,0.755)$ & $0.707(0.690,0.723)$ & $0.709(0.693,0.727)$ & $0.707(0.691,0.723)$ \\
\hline \multicolumn{5}{|c|}{ Intraclass correlation coefficient (\%) } \\
\hline Farm level & $55.7(49.9,60.5)$ & $58.1(51.9,58.7)$ & $58.3(52.4,58.1)$ & $58.2(52.4,58.6)$ \\
\hline Abattoir level & $15.4(5.9,25.5)$ & $11.1(7.3,15.4)$ & $10.7(8.4,15.2)$ & $10.8(7.9,14.7)$ \\
\hline \multicolumn{5}{|c|}{ Proportional change in variance (\%) } \\
\hline Farm level & - & $6.0(5.3,7.3)$ & $0.0(-0.5,0.8)$ & $0.4(0.2,0.7)$ \\
\hline Abattoir level & - & $35.3(27.3,41.5)$ & $4.0(1.7,6.2)$ & $-0.9(-2.3,1.6)$ \\
\hline Residual & - & $4.0(3.2,5.0)$ & $-0.2(-0.7,0.2)$ & $0.2(0.0,0.3)$ \\
\hline
\end{tabular}

The values enclosed by parentheses express the $95 \%$ confidence interval.

Model $0=$ only spatial non-parametric terms and random effects.

Model $1=$ Model $0+$ Production and management practices.

Model $2=$ Model $1+$ Soil characteristics.

Model 3 = Model $2+$ Climatic conditions before slaughter.

estimate of the between-farm prevalence was higher than described by Sanchez-Vazquez et al. (2010), who observed that $67.0 \%$ of the farms were infected. Note that the work carried out by Sanchez-Vazquez et al. is based on abattoir data collected by trained swine veterinarians who inspect every other pig on the slaughter line from a representative sample of each batch of pigs. Not every batch of pigs is eligible, only those that belong to the British abattoir monitoring health Schemes Wholesome Pigs Scotland (WPS) and British Pig Health Scheme (BPHS). This feature may explain some of the differences between their results and those of the present study.

We observed that the farms that showed some evidence of having a more intensified production system, according to the higher number of animals sent to slaughter, were at reduced risk of milk spots. These farms most likely have the protective housing conditions (e.g. floor type) and management practices (e.g. cleaning and disinfection procedures). According to Sanchez-Vazquez et al. (2010) the farms that (i) have buildings with solid floor and use bedding, (ii) have a dry feeding system, (iii) are just finishing units (not registered as breeding herds as well), and, (iv) have outdoor production stages are at increased risk compared with the opposite factors.

The inclusion of a socioeconomic indicator was helpful in complementing the scarce information regarding the farming conditions, which are critical in the epidemiology of the disease (Alban et al., 2015). It was hypothesised that more socioeconomically deprived farmers (i) may be less likely to apply preventive and control strategies to tackle the disease, (ii) may have weaker biosecurity structures and procedures in place, (iii) may produce pigs in conditions that better reflect external environmental pressures, (iv) may be less prone to seek veterinary advice, and (v) may be less likely to engage with major multi-company organisations. The use of a socioeconomic index to assign a particular deprivation level to the farms is not free of bias; however, it is reasonable to consider that the social context in which the farms are run (e.g. the level of education of the workers who provide assistance or the sanitary welfare of the neighbourhood) may have some effect. Curiously, a higher risk of milk spots among the farms located in poorer areas was observed at univariable analysis as well as at multivariable analysis without spatial non-parametric terms. This association became non-significant at the final model with spatial adjustment, which may be related to the high level of socio-spatial segregation existing in England (Musterd, 2005), i.e., EIMD15 is highly spatially autocorrelated. Thus, any adjustment for unmeasured spatial confounding (through the inclusion of smoothing splines for the longitude and latitude of the farm) inevitably leads to an attenuation of the effect of area socioeconomic deprivation.

This study also found interesting and new results regarding the influence of the type of soil on the proportion of livers rejected due to milk spots. According to the final model, the frequency of the disease is lower in peat soils, compared to coarse soils. Peat is a hydrogeomorphic soil that results from the decomposition of plants and mosses under wet conditions (Andriesse, 1988). In England, the hydrogeomorphic soils can occur in the form of hill or acid peat (formed in areas of high rainfall - e.g. the Pennine Chain) and fen or basin peat (formed in areas with excessive ground water - e.g. Yorkshire, Lincolnshire, and Norfolk) (Shirlaw, 2013). Coarse-textured soils $(18 \%<$ clay and $>65 \%$ sand) are characterised by low total pore space compared to fine-textured soils $(35 \%<$ clay $<60 \%)$. Under experimental conditions, soils that allow migration of the larvae appear to allow the geohelminths to find the best conditions of moisture and oxygenation (Beaver, 1953). Additionally, it has been reported that $A$. suum eggs survive longer when buried in soils that retain moisture, as it is the case of fine-textured soils (Mizgajska, 1993). The same has been suggested for A. lumbricoides: "clay soils favour Ascaris survival by retaining water around coated eggs and allowing them to withstand desiccation (...) and eggs mixed with clay adhere better to persons and clothing, increasing the chances for ingestion" (Spittell and Volpé, 1986;). Contrarily, our results do not suggest any biological advantage of $A$. suum in fine-textured soils and, indeed, reveal the limited impact of soil characteristics on the epidemiology of the disease.

Although extremely resistant in the environment, the eggs of $A$. suum do not develop at temperatures below $5{ }^{\circ} \mathrm{C}$ and its life cycle is highly dependent on humidity (Arene, 1986; Kim et al., 2012). This has been suggested as a possible reason for seasonal patterns of milk spots. Slow or no embryonation of eggs is common when they are excreted in the late autumn/winter until spring (Connan, 1977). Indeed, we observed a positive association between relative humidity, mean air temperature, and rainfall and the proportion of liver rejections due to milk spots. These results demonstrate the inherent effect of climatic conditions in swine production, even though it has become a highly industrialised sector.

The computation of variance components for the LMM showed that most of the variance occurs at farm level; this stresses the influence that farm-related factors have on the prevalence of the infection. The abattoir level also has a significant contribution but with much lower impact than the previous level, suggesting that differences in inspection procedures at the abattoir level could have a low impact on the prevalence estimates. That contribution was attenuated by the inclusion of block 1 (production and management practices - throughput and area socioeconomic deprivation), which highlights the relevance of stockmanship in the epidemiology of the disease. Soils characteristics and climatic conditions proved to be less relevant factors compared to the farm conditions. 


\subsection{Study strengths and limitations}

This study analysed the results of post-mortem inspection of 2,513,973 pigs from 1463 farms, which corresponds to approximately $29.5 \%$ of the total number of pigs slaughtered in 2014 in England ( $n=8,517,000)$ and $14.6 \%$ of all British pig farms $(n=10,000)$ (Department for Environment, Food and Rural Affairs, 2016). According to data from the Agriculture and Horticulture Development Board, $92 \%$ of the production comes from about 1600 farms, including ten corporate companies which account for $35 \%$ of breeding sows (AHDB, 2016). Although this study enrolled a considerable number of these farms (and pigs), the extent to which the results of this study can be generalised to the population of English farms is unknown.

The methodology used in this study has never been used in the context of porcine ascariasis, and it has a great potential to be applied in other conditions diagnosed at post-mortem inspection. In addition to the novelty of the methodology, we provide an update on the prevalence and spatial distribution of porcine ascariasis in England. Sanchez-Vazquez et al. had published the last prevalence results in 2012, when his team investigated the trend of milk spot lesions from July 2005 to December 2010. Their study used Pig Health Scheme records collected by trained veterinarians who inspect every second pig in a batch (up to fifty pigs assessed). In our study, we used official information gathered by meat inspectors who, working on behalf of the Food Standards Agency, inspect all the pigs slaughtered. While the fiveyear monitoring programme found evidence of $A$. suum infection at slaughter in $4.2 \%$ of 34,168 pigs inspected, our study did it in $4.3 \%$ of 2,513,973 pigs slaughtered in 2014.

This study is a pioneer in identifying a possible association between the socioeconomic profile of the farm's neighbourhood and the burden of swine ascariasis, highlighting the complex influence of social factors on the epidemiology of animal diseases.

The epidemiology of the disease is also remarkably related to environmental factors, in particular, the climatic conditions. However, farming related features should not be neglected because, as shown by the variance components analysis, they constitute the core of the studied epidemiological model. Thus, the fact that the majority of the farms had an unknown production system in place represents one of the most important caveats of the study.

We also observed that a significant proportion of the variance in the percentage of milk spots per batch was at abattoir level (approximately $15 \%)$. This result shows that official post-mortem inspection results need to be treated cautiously. The influence of (i) the geographical position of the premises, (ii) the specificities of the industry, and (iii) the standards of meat inspection are reasonable sources of bias. In a study that intended to assess the validity of traditional post-mortem inspection procedures for the detection of a chronic pathology in pigs, Enoe et al. (2003) argued that this kind of data should be carefully validated with double-classification of samples because the sensitivity of the traditional method was significantly lower than the extended one (designed for that specific purpose). This is, of course, another caveat of the present study, but it should also be stressed that we used information collected by fully qualified meat inspectors that follow the highest standards and state of the art procedures. Any inaccuracy that resulted in misclassification is unlikely and non-differential. This means that, in case of inaccuracy, the effect on risk estimates might have been diluted.

Although we added splines to account for the spatial dependency of observations, our approach might not capture subtle small-area variations in the outcome. For that, spatial Bayesian models would be more suitable (Dasgupta et al., 2014). Nevertheless, at NUT level, we found that observed and predicted values were very similar, suggesting that our approach is adequate to model this outcome at regional level.

Finally, the relevance of the postcode should be addressed carefully. Note that this was the unique parameter used for georeferencing the farms and this step may have added some information bias. First of all, compared to urban areas, rural areas (where farms are usually located) are less accurately georeferenced by postcode because each postcode unit comprises a larger territory. Secondly, providing a correct postcode, or even having a knowledge of it, may be more frequent among the farmers that have a higher education level, comply with the best farming practices and, thus, have fewer animals infected by A. suum.

\section{Conclusion}

Ascariasis is still an endemic disease of English farmed pigs. Although the number of animals infected per herd is relatively small, few farms are entirely free. The epidemiology of the disease is substantially related to environmental factors, including geohydrological characteristics of the soil as well as climatic features. However, stockmanship related issues should not be neglected because they constitute the core of the studied epidemiological model. Acknowledging these intermingled factors may support the development of new strategies to control the disease and guide the application of intervention measures by the industry.

\section{Conflict of interest statement}

The authors of this manuscript do not have any affiliations with or involvement in any organisation with a financial interest in the subject matter or materials discussed in this manuscript.

\section{Acknowledgements}

The authors are most grateful to all the institutions that released data used in this study, namely the European Soil Data Centre, Ordnance Survey, Met Office, Eurostat, Office for National Statistics and The National Archives (UK). We are also thankful for the support provided by the Food Standards Agency throughout the study, in particular by Gemma Forrest, since the moment post-mortem inspection data was shared.

This study was funded by FEDER through the Operational Programme Competitiveness and Internationalization and national funding from the Foundation for Science and Technology - FCT (Portuguese Ministry of Science, Technology and Higher Education) under the Unidade de Investigação em Epidemiologia - Instituto de Saúde Pública da Universidade do Porto (EPIUnit) (POCI-01-0145-FEDER-006862; Ref. UID/DTP/04750/ 2013).

\section{Appendix A. Supplementary data}

Supplementary data associated with this article can be found, in the online version, at http://dx.doi.org/10.1016/j.prevetmed.2017.09.012.

\section{References}

Agriculture and Horticulture Development Board (AHDB), 2016. Pig Production. http:// pork.ahdb.org.uk/pig-production Assessed on 23rd February 2016.

Alban, L., Petersen, J.V., Busch, M.E., 2015. A comparison between lesions found during meat inspection of finishing pigs raised under organic/free-range conditions and conventional, indoor conditions. Porcine Health Management 1 (1), 1-11.

Andriesse, J.P., 1988. Nature and management of tropical peat soils. FAO soils bulletin 59. Soil Resources, Management and Conservation Service. FAO Land and Water Development Division. Food and Agriculture Organisation of the United Nations, Rome, Italy.

Arene, F.O.I., 1986. Ascaris suum: Influence of embryonation temperature on the viability of the infective larva. J. Therm. Biol. 11 (1), 9-15.

Bates, D., Maechler, M., Bolker, B., Walker, S., 2015. Fitting linear mixed-effects models using lme4. J. Stat. Softw. 67 (1), 1-48.

Beaver, P.C., 1953. Persistence of hookworm larvae in soil. Am J. Trop. Med. Hyg. 2 (1), $102-108$.

Bernardo, T.M., Dohoo, I.R., Ogilvie, T., 1990. A critical assessment of abattoir surveillance as a screening test for swine ascariasis. Can. J. Vet. Res. 54 (2), 274-277.

Brooker, S., Clements, A.C., Bundy, D.A., 2006. Global epidemiology, ecology and control of soil-transmitted helminth infections. Adv. Parasitol. 62, 221-261.

Canty, A., Ripley, B., 2016. Boot: Bootstrap R (S-Plus) Functions R Package Version 1. pp. 3-18.

Chesworth, W., 2007. Field pH. In: Chesworth, W. (Ed.), Encyclopedia of Soil Science. 
Springer, Dordrecht, The Netherlands p. 271.

Connan, R.M., 1977. Ascariasis: the development of eggs of Ascaris suum under the conditions prevailing in a pig house. Vet. Rec. 100 (20), 421-422.

Dasgupta, P., Cramb, S.M., Aitken, J.F., Turrell, G., Baade, P.D., 2014. Comparing multilevel and Bayesian spatial random effects survival models to assess geographical inequalities in colorectal cancer survival: a case study. Int. J. Health Geogr. 13 (1), 36.

Department for Communities and Local Government, 2015. The English Indices of Deprivation 2015. Technical Report. . Retrieved from https://www.gov.uk/ government/uploads/system/uploads/attachment_data/file/464485/English Indices_of_Deprivation_2015_-_Technical-Report.pdf on 15th March 2016.

Department for Environment, Food and Rural Affairs, 2016. UK Monthly Numbers of Livestock Slaughtered. Retrived from https://data.gov.uk/dataset/slaughter statistics/resource/0b9aea62-5a94-42cb-ab0a-6b230dc763a4 on 27th May 2016.

Enoe, C., Christensen, G., Andersen, S., Willeberg, P., 2003. The need for built-in validation of surveillance data so that changes in diagnostic performance of post-mortem meat inspection can be detected. Prev. Vet. Med. 57 (3), 117-125.

Gerwert, S., Failing, K., Bauer, C., 2004. Husbandry management worm control practices and gastro-intestinal parasite infections of sows in pig-breeding farms in Munsterland. Germany Deutsche Tierärztliche Wochenschrift 111 (10), 398-403.

Gunawardena, G.S., Karunaweera, N.D., Ismail, M.M., 2004. Wet-days: are they better indicators of Ascaris infection levels? J. Helminthol. 78 (4), 305-310.

Kim, M.K., Pyo, K.H., Hwang, Y.S., Park, K.H., Hwang, I.G., Chai, J.Y., Shin, E.H., 2012. Effect of temperature on embryonation of Ascaris suum eggs in an environmental chamber. Korean J. Parasitol. 50 (3), 239-242.

Lai, M., Zhou, R.Q., Huang, H.C., Hu, S.J., 2011. Prevalence and risk factors associated with intestinal parasites in pigs in Chongqing, China. Res. Vet. Sci. 91 (3), e121-124.

Lal, R., 2006. Encyclopedia of soil science, Boca Raton. CRC Press, Taylor and Francis, Florida, USA.

McCormick, B.J., Sanchez-Vazquez, M.J., Lewis, F.I., 2013. Using Bayesian networks to explore the role of weather as a potential determinant of disease in pigs. Prev. Vet. Med. 110 (1), 54-63.

Menzies, F.D., Goodall, E.A., Taylor, S.M., 1994. The epidemiology of Ascaris suum infections in pigs in Northern Ireland, 1969-1991. Br. Vet. J. 150 (2), 165-172.

Merlo, J., Chaix, B., Yang, M., Lynch, J., Rastam, L., 2005. A brief conceptual tutorial of multilevel analysis in social epidemiology: linking the statistical concept of clustering to the idea of contextual phenomenon. J. Epidemiol. Commun. Health 59 (6), 443-449.

Mizgajska, H., 1993. The distribution and survival of eggs of Ascaris suum in six different natural soil profiles. Acta Parasitol. 38, 170-174.

Musterd, S., 2005. Social and ethnic segregation in Europe; levels: causes and effects. J. Urban Aff. 27, 331-348.

Neumann, E., Hall, W., Stevenson, M., Morris, R., Ling Min Than, J., 2014. Descriptive and temporal analysis of post-mortem lesions recorded in slaughtered pigs in New Zealand from 2000 to 2010. N. Z. Vet. J. 62 (3), 110-116.
Office for National Statistics, 2011. Lower Layer Super Output Area (LSOA) Boundaries for England and Wales. Retrieved from https://data.gov.uk/dataset/lower_layer super_output_area_lsoa_boundaries on 6th November 2015.

Roepstorff, A., Nansen, P., 1994. Epidemiology and control of helminth infections in pigs under intensive and non-intensive production systems. Vet. Parasitol. 54 (1-3), 69-85.

Roepstorff, A., Nansen, P., 1998. Epidemiology, Diagnosis and Control of Helminth Parasites of Swine. Food and Agriculture Organisation of the United Nations, Rome, Italy.

Sánchez Thevenet, P., Nancufil, A., Oyarzo, C.M., Torrecillas, C., Raso, S., Mellado, I. Flores, M.E., Cordoba, M.G., Minvielle, M.C., Basualdo, J.A., 2004. An eco-epidemiological study of contamination of soil with infective forms of intestinal parasites. Eur. J. Epidemiol. 19 (5), 481-489.

Sanchez-Vazquez, M.J., Smith, R.P., Kang, S., Lewis, F, Nielen, M., Gunn, G.J, Edwards, S.A., 2010. Identification of factors influencing the occurrence of milk spot livers in slaughtered pigs: a novel approach to understanding Ascaris suum epidemiology in British farmed pigs. Vet. Parasitol. 173 (3-4), 271-279.

Sanchez-Vazquez, M.J., Strachan, W.D., Armstrong, D., Nielen, M., Gunn, G.J., 2011. The British pig health schemes: integrated systems for large-scale pig abattoir lesion monitoring. Vet. Rec. 169 (16), 413.

Sanchez-Vazquez, M.J., Nielen, M., Gunn, G.J., Lewis, F.I., 2012. National monitoring of Ascaris suum related liver pathologies in English abattoirs: a time-series analysis, 2005-2010. Vet. Parasitol. 184 (1), 83-87.

Schüle, S.A., Clowes, P., Kroidl, I., Kowuor, D.O., Nsojo, A., Mangu, C., Riess, H., Geldmacher, C., Laubender, R.P., Mhina, S., Maboko, L., Löscher, T., Hoelscher, M., Saathoff, E., 2014. Ascaris lumbricoides infection and its relation to environmental factors in the Mbeya Region of Tanzania, a cross-sectional, population-based study. PLoS One 9 (3), e92032.

Shirlaw, D.W.G., 2013. Factors affecting the choice of farming systems: soils. In: Shirlaw, D.W.G. (Ed.), An Agricultural Geography of Great Britain. Pergamon Press Ltd., Oxford, UK, pp. 10-20.

Shukla, M.K., 2013. Characteristics of soils of the Vadose Zone. In: Shukla, M.K. (Ed.), Soil Physics: an Introduction. Boca Raton. CRC Press, Taylor and Francis, Florida, USA, pp. 20-57.

Spittell, J.A., Volpé, R., 1986. Ascaris lumbricoides epidemiology. In: In: Spittell, J.A. Volpé, R. (Eds.), Clinical Medicine, vol. 3 Harper and Row, New York, USA p. 122.

Stewart, T.B., Hoyt, P.G., 2013. Internal parasites. In: Straw, B.E., Zimmerman, J.J., D'Allaire, S., Taylor, D.J. (Eds.), Taylor Diseases of Swine. Blackwell Publishing, Oxford, UK, pp. 901-914.

Valencia, L.I., Fortes, P., Medronho, A., 2005. Spatial ascariasis risk estimation using socioeconomic variables. Int. J. Environ. Health Res. 15 (6), 411-424.

Yoshihara, S., Nakagawa, M., Suda, H., Ikeda, K., Hanashiro, K., 1983. White spots of the liver in pigs experimentally infected with Ascaris suum. Natl. Inst. Anim. Health Q. (Tokyo) 23 (4), 127-137. 